\title{
Extrusion of Endodontic Filling Materials: Medico-Legal Aspects. Two Cases
}

\author{
Valeria Santoro ${ }^{1, *}$, Piercarlo Lozito ${ }^{2}$, Antonio De Donno ${ }^{3}$, Felice Roberto Grassi ${ }^{4}$ and \\ Francesco Introna $^{5}$
}

${ }^{1}$ Section of Legal Medicine, University of Bari, Italy; ${ }^{2}$ Section of Legal Medicine, University of Bari, Italy; ${ }^{3}$ Section of Legal Medicine, University of Bari, Italy; ${ }^{4}$ Department of Odontostomatology and Surgery, University of Bari, Italy and ${ }^{5}$ Section of Legal Medicine, University of Bari, Italy

\begin{abstract}
The Authors describe two cases of alleged malpractice due to overfilling. The aim of this article is to underline some medico-legal aspects regarding the quantity of extruded material which may be considered acceptable and the consequent damage to the patient.

Two cases are presented here: In the first case, the dentist's liability is clear due to excessive extrusion of endodontic material beyond the apical region combined with incomplete obturation of the canals. In the second case however, because two different dentists were involved, establishing the connection of causality between their work and the damage reported by the patient was not easy. This situation makes it difficult to establish the limits of potential responsibility, coupled with the complete absence of radiographic signs of periapical rarefaction and the small quantities of material beyond the apex. From a medico-legal point of view, a dentist may be held responsible for compensation and financial expenses of a patient for restoration of damage resulting from a dental procedure.

Italian guidelines offer no indications as to when overfilling should be considered the result of a procedural error, or if it fits within the range labelled as "acceptable" and this gap offers extremely subjective interpretations of legal consultants.

So, it would therefore be useful to adopt more precise qualitative/dimensional parameters, keeping in mind that the guidelines offer therapeutic recommendations and are not rigid protocols.
\end{abstract}

Key Words: Overfilling, professional liability, endodontic procedurals errors, forensic dentistry.

\section{INTRODUCTION}

For some Authors the use of techniques which use vertical condensation of warm gutta-percha during the obturation phase offer a higher probability of closure of the lateral and accessory canals $[1,2]$. At the same time however, warm vertical compaction techniques also result in a greater risk of the obturation material being extruded into periradicular tissues [3-5]. This material, usually cement, acts as a lubricant, as it aids in the progression of the principal obturation material (core) during the compaction phase. Furthermore, it also aids in the filling of the lateral and accessory canals which would otherwise be impossible to fill with a single core of gutta-percha. In addition, it improves the adaptation to discrepancies and irregularities which, even after correct shaping, may persist on the root canal wall.

Beyond providing the ability of re-absorption on contact with tissue fluids, it is imperative that endodontic cements are biocompatible, and that they respect the periapical tissue. It is also preferable that they possess good antimicrobial properties.

In most cases irritation of the periapical tissues from extrusion of endodontic cement, is transitory with subsequent

\footnotetext{
*Address corrispondence to this author at the Section of Legal Medicine, University of Bari, Policlinico, Piazza Giulio Cesare 11. 70124, Bari, Italy; Tel/Fax: +390805478249/+390805478301; E-mail: vasantoro@virgilio.it
}

reabsorption of the excess material, leading to complete healing in a few months. This phenomenon may be considered to be an expected complication, and at times, even sought after by many dentists as a sign of a successfully completed intervention [6].

There is notable controversy in the literature, however, regarding the presence of cement beyond the apex. Some authors, among them Schilder (1967), refute the hypothesis that the presence of cement beyond the apex favours healing of the periapical lesions, maintaining their benign nature. $\mathrm{He}$ asserts that extrusion beyond the apex must be avoided solely in the interest of potential discomfort created for the patient during the obturation phase [7].

Other authors have reported significant cytotoxicity of both commonly used cements, and gutta-percha following research studies carried out in vitro with SEM (scanning electron microscope) [8]. This cytotoxicity can induce periradicular inflammation, or necrosis of the periodontal ligament, and for this reason overfilling should be avoided as much as possible because it can lead to failure of short term treatment or a negative long term prognosis [9-12].

The possibility of deterioration of these materials and the subsequent release of toxic substances over time, which can inhibit the proliferative capacity of some periradicular cell lines, have led some authors to assort that techniques which 
result in routine extrusion of cement beyond the apex are unsuitable [13-16].

In reality, the prognosis for an endodontically treated tooth with overfilling depends on the response of the periradicular tissue to the canal obturation material which is, in its own way, a consequence of the complex, and at times an unpredictable interaction between the materials and the host defences [17].

In accordance with the guidelines approved by Comitato Intersocietario di Coordinamento delle Associazioni Odontostomatologiche Italiane (CIC) in 2003, a small amount of endodontic material beyond the apex could be considered admissible only if it does not constitute an insult to vitally important structures, such as the inferior alveolar nerve and the maxillary sinus. Furthermore, in the presence of extrusion, and in the absence of post-operative pain, the dentist may want to consider reporting the existence of an endodontic "overfilling" in the patient's clinical file which might be able to explain the appearance of any possible future symptoms. However, when extrusion is present, if there are pain symptoms immediately following the therapy which persist with no apparent apical lesion, it may be appropriate to perform an orthograde re-treatment. And should the symptoms persist, surgical endodontics should be carried out as a last resort [18].

\section{CASE 1}

The first case studied concerns a 24 year old male who was experiencing pain in the first mandibular molars (36 and 46) which had been restored, but affected by secondary caries (Fig. 1).
He sought dental center where an orthopantomograph was taken, followed by root canal therapy, and restorations of both teeth. At the end of treatment, which lasted for about 4 months, persistent pain, particularly in tooth 36 , and widespread throughout the left side of the mandible, prompted the patient to seek out another dental practice where another orthopantomograph was taken and periapical lesions correlated to excessive extrusion of endodontic material beyond the apical region were diagnosed in both treated dental elements (Fig. 2). There was an associated mandibular swelling and a associated fistula deep abscess fistula of tooth 36 . Because of the amount of extruded material, the second dentist deemed the extraction of 36 necessary, and decided to start an orthograde retreatment of 46 . Tooth 36 was exported followed by alveolar curettage of the periradicular bone and orthograde endodontic retreatment of tooth 46. Further treatment included the insertion of an osseointegrated implant to replace tooth 36 , in light of the young age of the patient.

Once a connection between the conduct of the first dentist and the consequent damage was established, he was found to be responsible and was forced to pay for both the biological damage caused by the necessary extraction of tooth 36 by the second dentist, which was only partially able to be corrected by prosthetic-implant therapy, as well as the costs of all the other necessary subsequent treatments.

\section{CASE 2}

The second case concerns a patient who presented with painful symptoms corresponding to tooth the maxillary right lateral incisor. Following tests to determine pulp vitality and

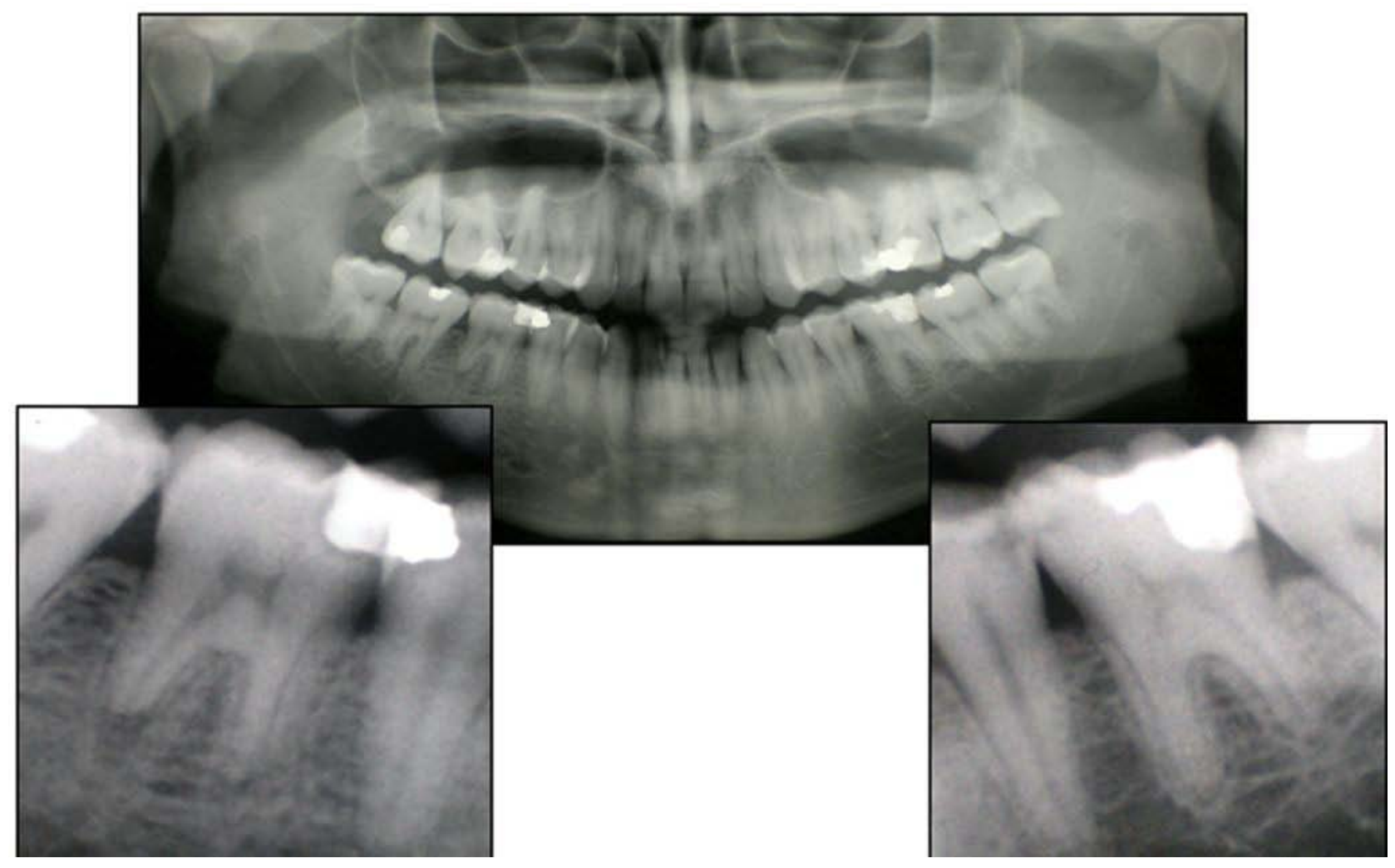

Fig. (1). Case 1. The ortopantomograph shows left and right first inferior molars (3.6-4.6) with secondary decay. 


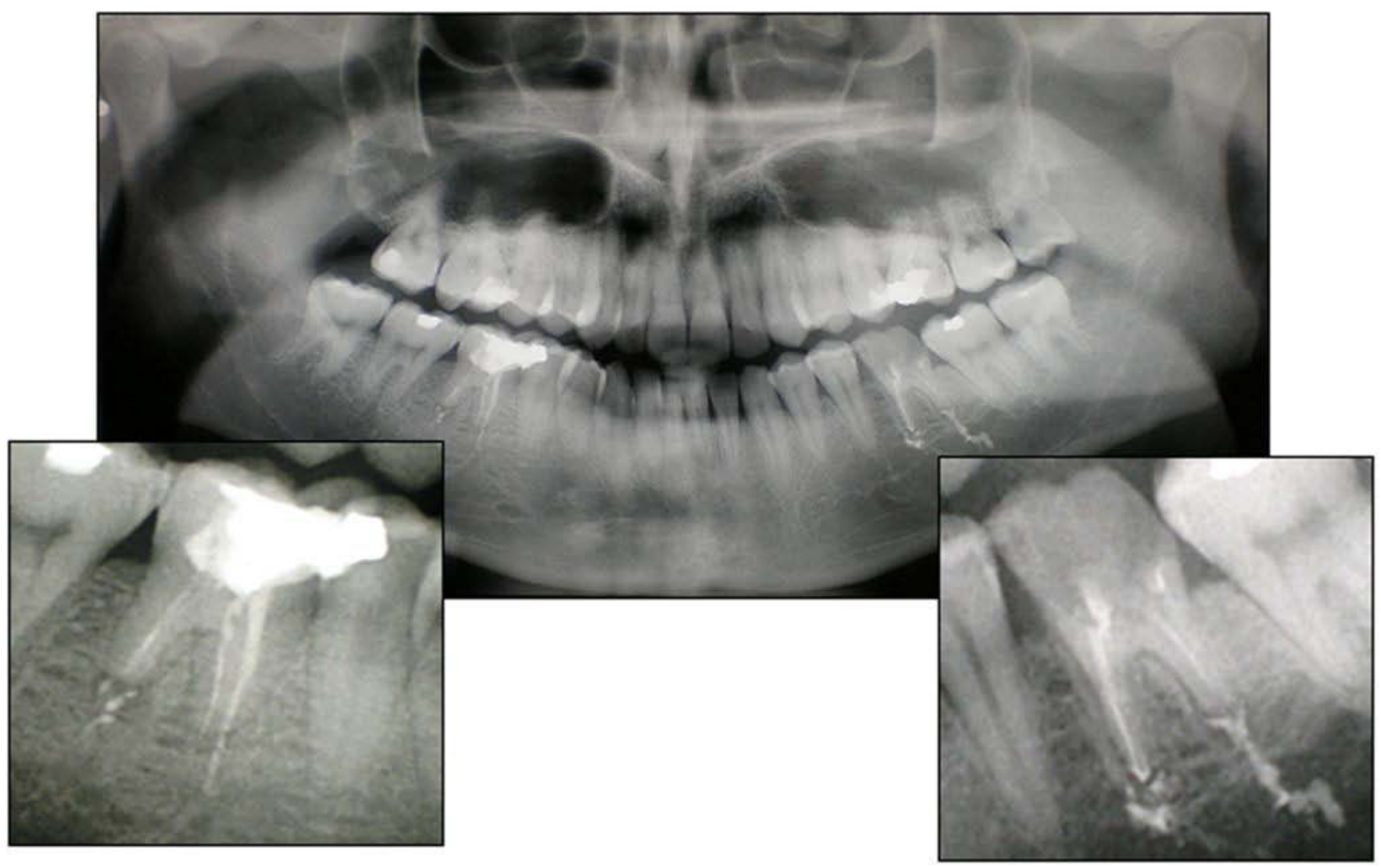

Fig. (2). Case 1. The second opg shows the excessive extrusion of endodontic material beyond the apical region and the incomplete obturation of the canals.

the absence of periodontal defects on radiological exam a diagnosis of acute pulpitis was made (Fig. 3). It was decided, therefore to begin canal therapy of the tooth concerned, followed by post-endodontic reconstruction. An orthograde endodontic treatment was carried out utilizing the warm vertical condensation technique (System B/manufacturer), with a cone of Fine-Medium gutta-percha, as well as zinc oxide/eugenol based endocanal cement (Pulp Canal Sealer/ manufacturer). At his 3 month check-up, the patient complained of pain when chewing in an area most likely coinciding with the apex of the treated tooth. Examination showed there was no evidence of an area of radio-lucency in the periapical region of tooth 12 during the radiological exam, but there was slight extrusion of obturation material beyond the apex (Fig. 4).

Clinical examination revealed the presence of wear facets of the crown ascribable to attrition, and so the patient was recommended for a gnatological visit. However it was decided to begin the retreatment of element 12 in order to carry out a wider shaping of the apical zone, along with prolonged irrigation in order to address any possible residual floral bacteria in the endodontic space which was responsible for the persistence of the inflammatory process [19].

The canal was closed and sealed for the second time with gutta-percha Medium and endocanal cement (Pulp Canal Sealer), using the warm vertical condensation technique. Radiological testing after another 3 months revealed no signs of periapical pathology, but the patient referred the persistence of pain (Fig. 5).

After having been contacted by the first practice after one year for a check-up, the patient informed them that he had attended another dentist because of the persistence of painful symptoms which corresponded to the periapical region of tooth 12 , and that he had undergone endodontic surgery.

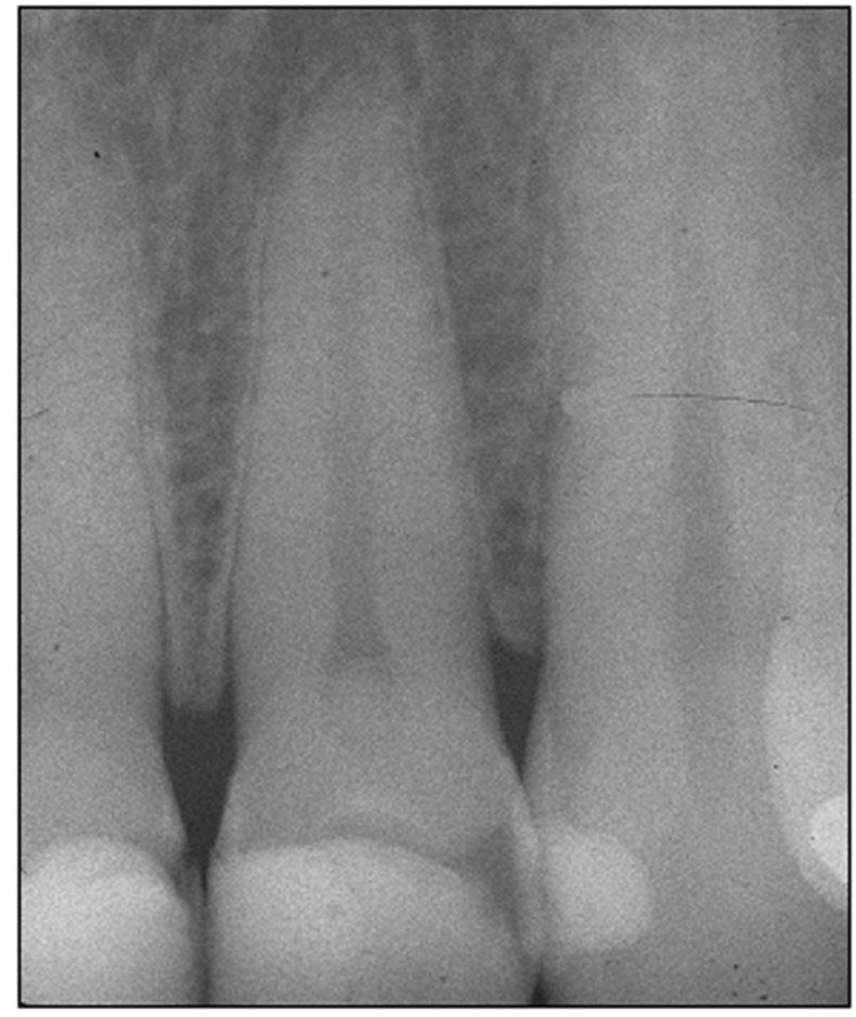

Fig. (3). Case 2. Pre-operative radiological exam of right lateral upper incisor (1.2) with secondary decay. 


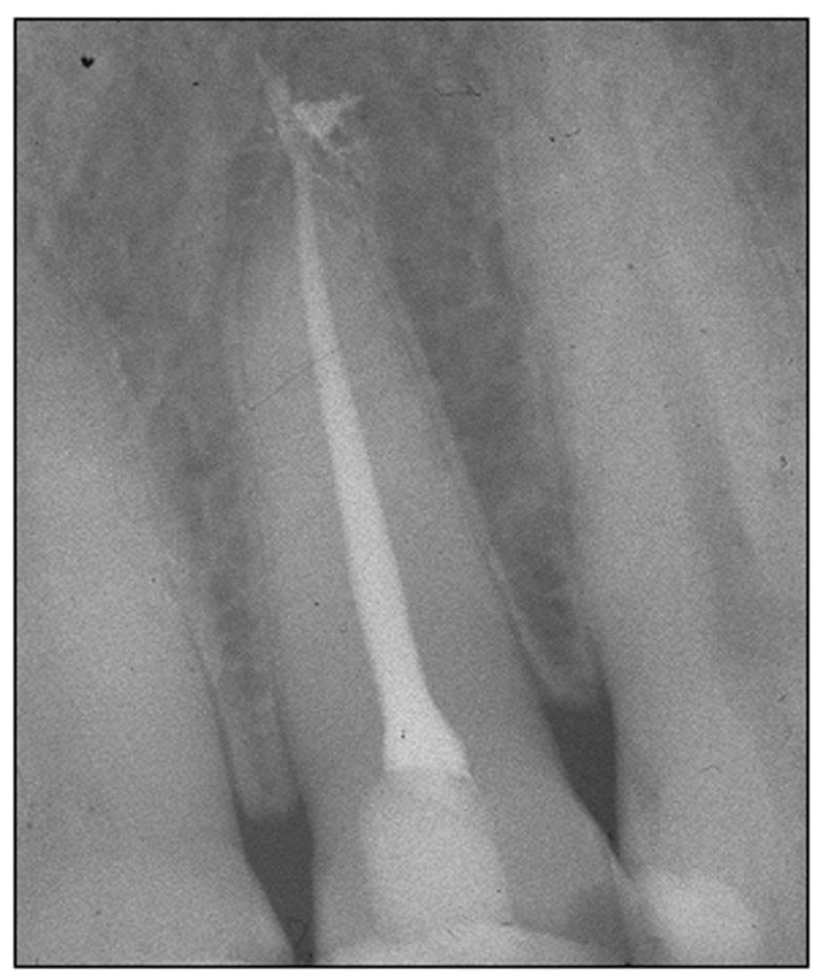

Fig. (4). Case 2. Radiological exam of 1.2 after the first orthograde endodontic treatment with warm vertical condensation technique and apical extrusion of sealer (first dentist).

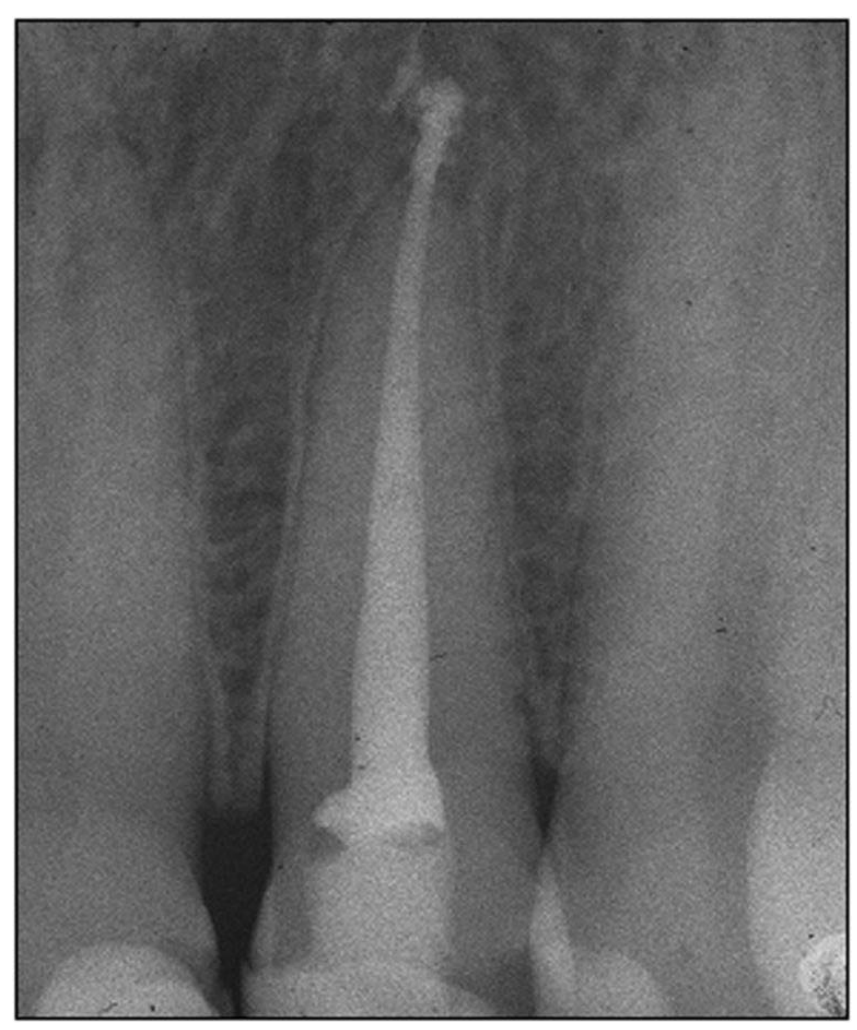

Fig. (5). Case 2. Radiological exam after the orthograde retreatment of 1.2 with warm vertical condensation technique and apical extrusion of sealer (first dentist).

Clinical examination revealed soreness upon percussion of the tooth and a semi lunar scar in the mucosa adjacent to tooth 12 . Radiological examination revealed a radiopacity at the apex of tooth 12 , suggested that an amalgam had been used as the root-end filling material. There was also an area of periapical radiolucency (Fig. 6).

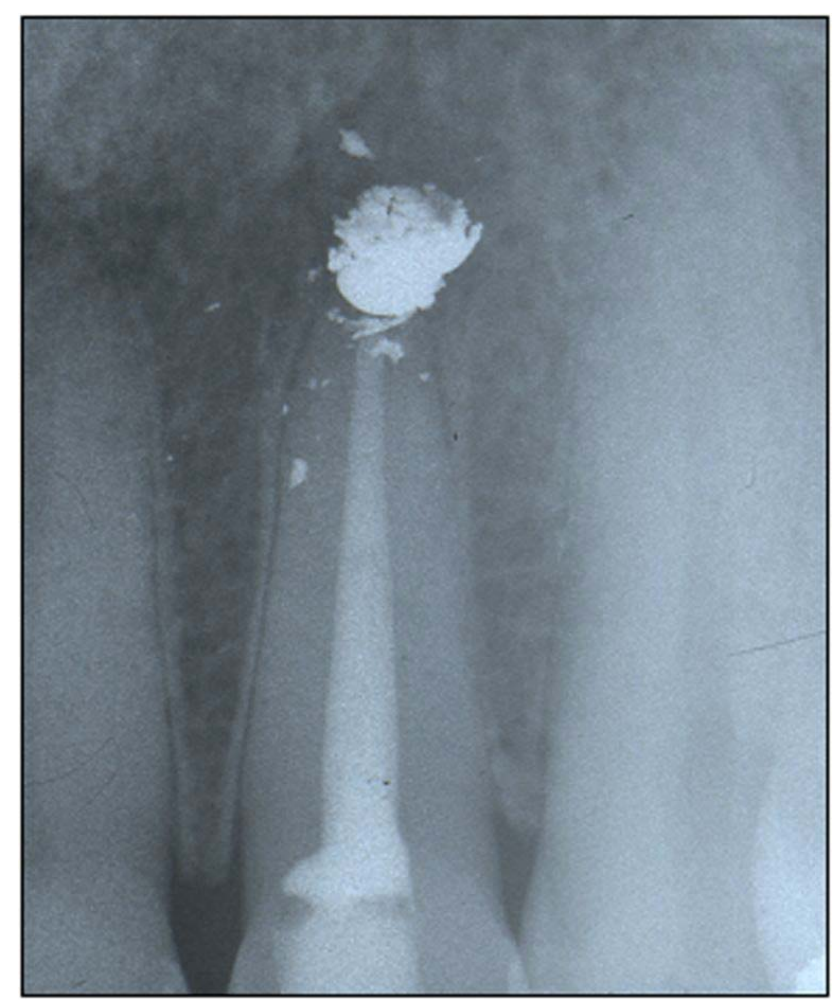

Fig. (6). Case 2. Radiological exam after the first endodontic surgery and retrograde obturation with amalgam of 1.2 (second dentist).

Two months later the first dentist performed a second surgical revision of the apex by means of an intrasulcular incision creating a flap by using Velvart's technique [20], osteotomy by ultrasonies, removal of the obturation material confirming the presence of amalgam and its scattered fragments in the bony cript. The prior resection of the apex was confirmed by the presence of a chamfer which was eliminated and followed by the preparation of the cavity using ultrasound to a depth of $3 \mathrm{~mm}$. The obturation material chosen was MTA (Fig. 7).

The patient did not attend for removal of the sutures, nor for subsequent check-ups, and a few months later, the dentist received notice from the patient's lawyers informing him of a lawsuit. The patient complained of worsening of the preexisting pain symptoms, as well as the occlusion of his right nostril with associated difficulty in breathing, ascribable to, according to the citation, the extrusion of endodontic material into the nasal cavity.

\section{DISCUSSION}

From a medico-legal point of view, there are basically two questions to ask: What is the quantity of extrusion material which may be considered acceptable?

According to the American Dental Association, overfilling by more than $2 \mathrm{~mm}$ past the radiological apex represents a technical error ascribable to over-instrumentation, inadequate measuring, or a lack of an apical stop. However the latter was difficult to obtain, as in the presence of resorbed 
roots caused by inflammatory processes or by particularly wide apices [14].

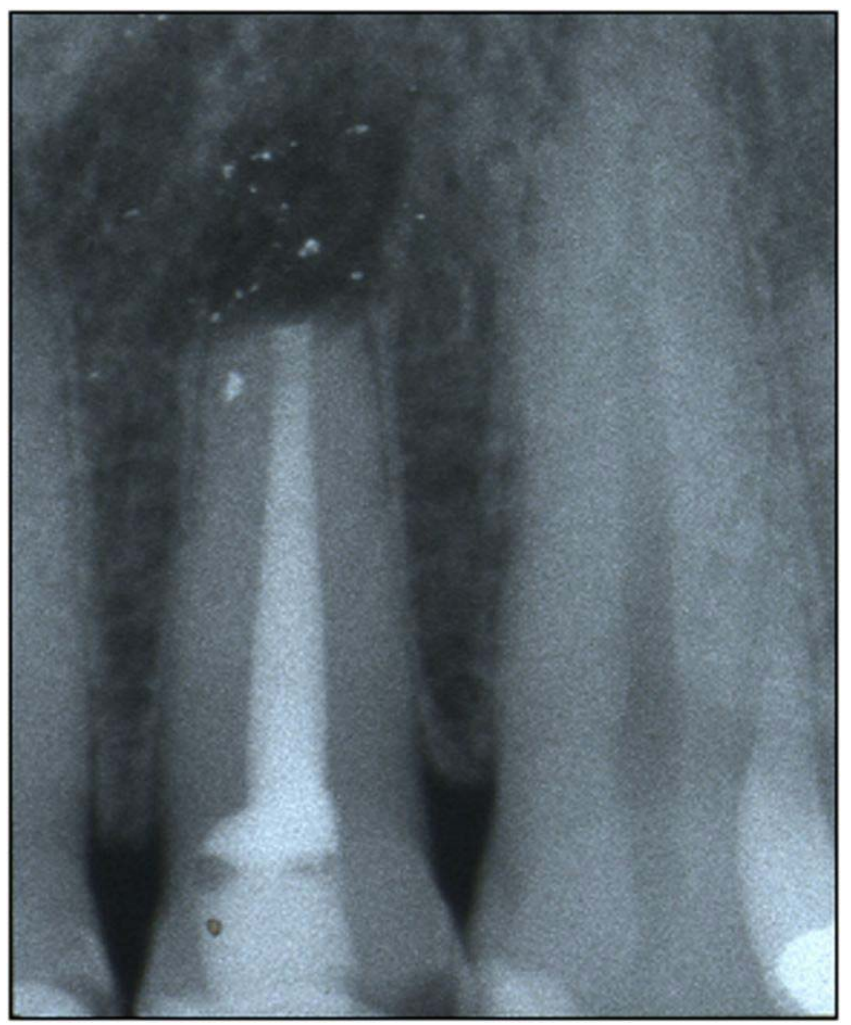

Fig. (7). Case 2. Radiological exam after the second apicoectomy and retrograde obturation with MTA of 1.2 (first dentist).

Overinstrumentation, in particular, may extrude infected material contained in the canals beyond the apex, interfering, or impeding the healing process of the periapical tissue. Gutta percha cones which had been extruded past the apices and subsequently examined under a scanning electron microscope, have demonstrated the presence of a "biofilm" on the cones [8]. This "biofilm" allows undisturbed growth of the bacteria and renders them particularly resistant to the defences of the host, and may be responsible for foreign body reactions. The consequences of overfilling can, therefore, result in infective periapical periodontitis caused by the transport of bacteria beyond the apex and an incomplete cleansing; foreign body reactions; and pain symptoms which are ascribable to irritative stimuli, even in the absence of radiological evidence [21-23].

In such cases, from a medico-legal point of view, the operator could be held responsible for the compensation and financial expenses for the treatment of the resulting damage, as well as for the temporary disability of the patient and the prolonging of treatment, which could involve either an orthograde root canal re-treatment, or the need for surgical intervention.

It is also necessary to specify that surgical endodontics may be limited by local anatomical factors such as an inaccessible root end, tooth with inadequate periodontal support, uncooperative patient or patient with a compromised medical history [24].

Even though, in certain cases it may be necessary to remove the tooth in question, this will result in damage due to the loss of the tooth, which would be only partially correctible using prosthetic substitution of the element.

Italian law states that in a contractual relationship between the patient and dentist: "the debtor (dentist) who does not accurately carry out a service is held responsible for damages if he is unable to demonstrate that fault or prolonged time was determined by the impossibility of the procedure (art. 1218 Civil Code)" [25].

Even when using the latest and best techniques for achieving the best results, as in the case of warm vertical condensation technique, the dentist is responsible for any possible risks connected with their application, as well as any errors made. It remains necessary, therefore, to establish the amount of overfilling allowed, in order to determine if an error has indeed occurred.

Different from other situations is the case of the invasion of material beyond the apex when critical anatomical structures, such as the maxillary sinus or the mandibular canal are affected, with consequential sinusitis or neurological damage from neurotoxicity or mechanical compression.

Under such circumstances, even without considering the amount of material extruded beyond the apex, assignment of guilt is unavoidable and may be due to negligent behaviour, arising from the neglect of common rules of diligence due to superficiality or irresponsibility, which in such cases may indicate the lack of an apical stop, or incorrect working lenght; or carelessness, if the damage is caused by recklessness or unjustified haste which impedes the use of proper precautions as in a case of adjacent elements of important anatomical structures whose lesions and their consequences must not be ignored by the dentist.

Also in this case the consequences will be the compensation for any biological damage if the invasion of critical anatomical structures results in a sickness, even after the elimination of excess material, only of course, if this is possible.

Different than the first case presented, in the second case recognising a connection between a dentist and the damage complained about by the patient is not such an easy task, because there was the work of two dentists, which make it difficult to establish the boundaries of possible responsibility, together with the complete absence of radiographic signs of periapical inflammation.

Nevertheless, as has already been underlined, it is necessary to keep under consideration reported symptomatology which leads to orthograde retreatment and two surgical operations.

Infact, in this case, there was a patient who presented with symptoms which required orthograde retreatment and two surgical interventions with unsatisfactory outcomes.

But it is also important to remember that there is a 70-90 $\%$ success rate in endodontic procedures and that there is always the possibility of unforeseen biological reactions.

Infact as with any medical intervention, the outcome depends on the biological variability of the individual, the ability of the dentist, or a combination of both. It is understood that even a "genuine" endodontic treatment can fail $[26,27]$.

Confirming the unequivocal interpretations regarding indications for treatment (pulpitis, pulpar necrosis, prosthetic 
treatment, etc.), and the criteria for defining success (absence of pain, absence of oedema, absence of fistulas, absence of lesions, well defined lamina dura, and restored functionality), there still remain some disagreements between the various schools of thought as to procedures and adopted techniques.

There is no agreement, in fact, regarding the radicular level at which the treatment should reach, even though some meta-analyses have recognised that, over time, the best results for canal obturations occur when the gutta-percha arrives at $0-1 \mathrm{~mm}$ from the apex and, on the contrary, when considering measurements of greater than $1 \mathrm{~mm}$ (above or below the apex), the results are less favourable [28].

For those gutta-percha compacting techniques which may involve an extrusion, there are no indications as to when overfilling should be considered the result of a procedural error, or if it fits within the range labelled as "acceptable".

\section{CONCLUSIONS}

It would be useful to use more precise qualitative/dimensional parameters, as in the case of the American Dental Association, which recognises the extrusion of material greater than $2 \mathrm{~mm}$ beyond the apex as an error in order to avoid vague indications which offer extremely subjective interpretations on the part of the legal consultants called to judge the conduct of colleagues in the medico-legal field, inevitably influencing the proceedings regarding professional responsibility.

Nevertheless, it must be kept in mind that the guidelines offer therapeutic recommendations and are not rigid protocols. Therefore, not strictly following them does not imply that the dentist is responsible. Especially in a courtroom situation, it must be remembered that each medical treatment must be scrupulously analyzed with respect to the subjective distinctiveness and the freedom of the dentist to choose the therapy which he thinks to be the best, obviously keeping in mind his patient's health.

\section{ACKNOWLEDGEMENT}

The authors would like to thank Dr. Giuseppe Carrieri for his collaboration in the preparation of this manuscript.

\section{REFERENCES}

[1] Dulac KA, Nielsen CJ, Tomazic TJ, et al. Comparison of the obturation of lateral canals by six techniques. J Endod 1999; 25(5): 37680 .

[2] Clinton K, Himel VT. Comparison of warm gutta-percha obturation technique and lateral condensation. J Endod 2001; 27(11): 692-5.

[3] Al-Dewani N, Hayes SJ, Howell Dummer PM. Comparison of laterally condensed and low temperature thermoplasticized guttapercha root fillings. J Endod 2000; 26(12): 733-8.

[4] Kececi AD, CelikUnal G, Sen BH. Comparison of cold lateral compaction and continuous wave of obturation technique following manual or rotary instrumentation. Int Endod J 2005; 38: 381-88.
[5] Peng L, Ye L, Tan H, et al. Outcome of root canal obturation by warm gutta-percha versus collateral condensations. A meta-anlysis. J Endod 2007; 33: 106-9.

[6] Nguyen TN. Obturation of the root canal system. In: Cohen S, Burns RC, Eds. Pathways of the pulp. $7^{\text {th }}$ ed. St Louis: Mosby Inc 1994; pp. 219-71.

[7] Schilder H. Filling root canals in three dimensions. Dent Clin North Am 1967: 723-44.

[8] Gutierrez JH, Brizuela C, Villota E. Human teeth with periapical pathosis after overstrumentation and overfilling of the root canals: a scanning electron microscopic study. Int Endod J 1999; 32: 40-8.

[9] Pascon A, Leonardo MR, Safovi K, et al. Tissue reactions to endodontic materials: criteria, assessment and and observations. Oral Surg Oral Med Oral Pathol Oral Radiol Endod 1991; 72: 222-37.

[10] Dahl JE. Toxicity of endodontics filling materials. Endod Top 2005; 12: 39-43.

[11] Ørstavik D. Materials used for root canal obturation technical, biological and clinical testing. Endod Top 2005; 12: 25-38.

[12] Ho YC, Huang FM, Chang YC. Mechanism of citotoxicity of eugenol in human osteoblastic cells in vitro. Int Endod J 2006; 39: 389-93.

[13] Yusuf H. The significance of presence of foreign material periapically as a cause of failure of root canal treatment. Oral Surg Oral Med Oral Pathol Oral Radiol Endod 1982; 54: 566-74.

[14] Lin LM, Rosenberg PA, Lin J. Do procedural errors cause endodontic treatment failure? J Am Dent Assoc 2005; 136: 187-93.

[15] Ektefaie MR, David AT, Poh CF. Surgical resolution of chronic tissue irritation caused by extruded endodontic filling material. J Can Dent Assoc 2005; 71(7): 487-90.

[16] Lee YY, Hung SL, Pai SF, et al. Eugenol suppressed the expression of lipopolysaccharide induced proinflammatory mediators in human macrophages. J Endod 2007; 33:698-702.

[17] Sjögren U, Figdor D, Persson S, et al. Influence of infection at the time of root filling on the outcome of endodontic treatment of teeth with apical periodontitis. Int Endod J 1997; 30: 297-306.

[18] Comitato Intersocietario di Coordinamento delle Associazioni Odontostomatologiche Italiane (CIC). Linee Guida in Odontoiatria. Firenze 2003; pp. 73-8.

[19] Bystrom A, Happonen RP, Syogren U, et al. Healing of periapical lesions of pulpless teeth after endodontic treatment with controlled asepsis. End Dent Traumatol 1987; 3(2): 58-63.

[20] Velvart P. Papilla base incision: a new approach to recession-free healing of the interdental papilla after endodontic surgery. Int Endod J 2002; 35(5): 453-60.

[21] Bergenholtz G, Lekholm U, Milthon R, et al. Influence of apical overinstrumentation and overfilling on re-treated root canals. J Endod 1979; 5: 310-14.

[22] Holland R, De Souza V, Nery MJ, et al. Tissue reactions following apical plugging of the root canal with infected dentin chips. Oral Surg Oral Med Oral Pathol Oral Radiol Endod 1980; 49: 366-9.

[23] Costerton JW, Stewart PS, Gerenberg EP. Bacteria biofilms: a common cause of persistent infections. Science 1999; 284: 131824.

[24] Lost C. Quality guidelines for endodontic treatment: consensus report of the European Society of Endodontology. Int Endod J 2006; 39: 921-30.

[25] Puccini C. Istituzioni di medicina legale. 6th Milano Casa Editrice Ambrosiana 2003; pp. 980-3.

[26] Siqueira JF Jr. Aetiology of root canal treatment failure :why welltreated teeth can fail. Int Endod J 2001; 34(1): 1-10.

[27] Torabinejad M, Lemon RR. Procedural accidents. In: Walton R, Torabinejad M, Eds. Principles and practice of endodontics. $3^{\text {rd }}$ ed. Philadelphia Saunders 2002; pp. 310-30.

[28] Schaeffer MA, White RR, Walton RE. Determining the optimal obturation length: a meta-analysis of literature. J Endod 2005; 31: 271-4. 